\title{
Independent motion segmentation and collision prediction for road vehicles
}

\author{
D. Sinclair, B. Boufama, R. Mohr \\ LIFIA-INRIA, \\ 46 Av. Félix Viallet, F- 38031 Grenoble Cedex, France
}

\begin{abstract}
This paper presents a method for doing motion segmentation for autonomous vehicles which drive on planar surfaces. There are two distinct types of independent motion that may occur within an image sequence taken from a moving vehicle. The first generic type of independent motion is when the projected motion of points on the independent object violate the epipolar constraint. The second case is where the epipolar constraint is not violated. This paper demonstrates that it is possible to detect this second type of independent motion by looking for progressive dis-occlusion of the road. A novel collision prediction method is also given. The method predicts the projection of a corridor down which the AGV will travel. This prediction may be used for time to contact collision prediction and the corridor width embodies an estimate of the vehicles size.
\end{abstract}

Keywords: Motion segmentation, occlusion, collision avoidance.

\section{Introduction}

This paper tackles an important problem currently outstanding in robot vision, that of independent motion segmentation. If robots are to interact with unknown or unpredictable environments then they must be able to determine which part of a scene is moving independently and therefore should be payed attention to.

There are two distinct types of independent motion as far as robot vision is concerned. The first is where the independently moving object is not moving parallel to the camera on the AGV. In this case there will be two distinct epipoles, one for the camera motion relative to the background and the other for the camera motion relative to the moving object. The second type of independent motion is where the other object is moving in the same direction as the camera. In this case the epipolar constraint will not be violated An example of this kind of motion would be an AGV driving down a straight road following another vehicle. The only way to detect such motion is to look at the occluding boundary of the second vehicle. This paper presents robust solutions for finding both these types of independent motion in a road vehicle environment.

There exist two distinct approaches to motion segmentation. The first relies on knowing or partially knowing the translation $[2,9,8,7]$. Existing structure from motion algorithms $[6,4,3]$ might be used to flag the presence of independent motion by looking at the error terms in the least squares minimisation involved

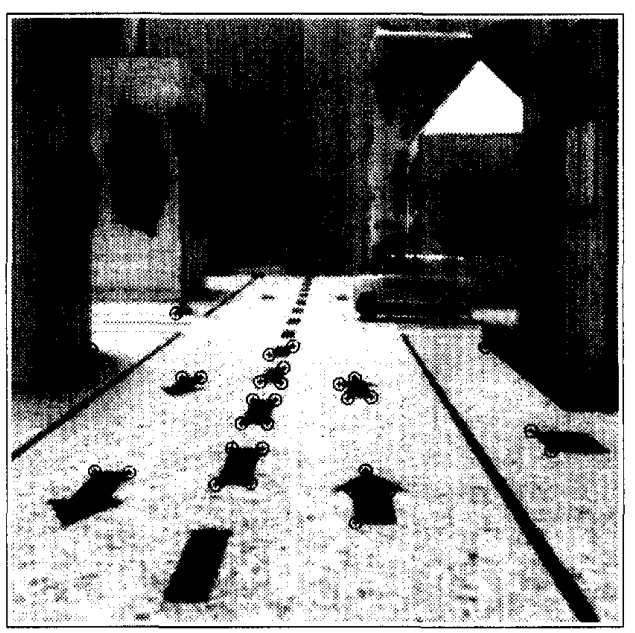

a)

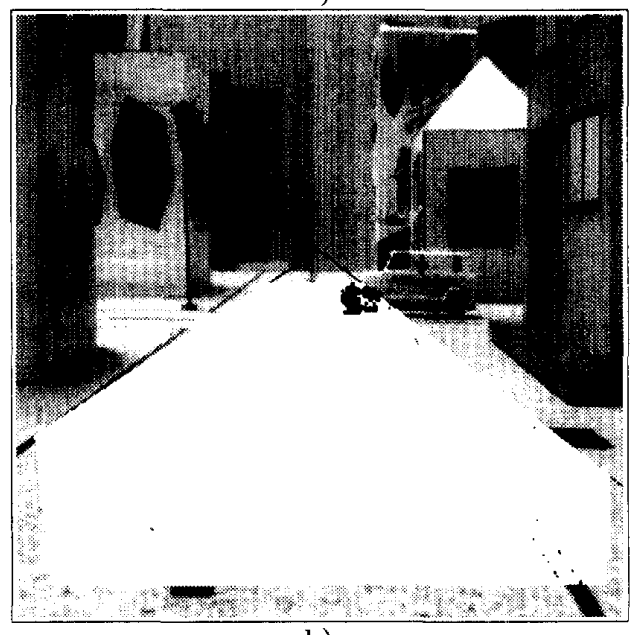

b)

Figure 1: a) tracked corners found to be on the plane of the road are marked with $\oplus$ all other tracked corners are marked with +signs. b) the dense drivable region is coloured white. 
in their implementation but these errors might also have resulted from outliers in the data. This would effectively be a regression fitting method and is likely to suffer from convergence problems if a good initial guess at the motion direction is not available.

The second approach is to assume a local model for the flow field and apply robust statistics $[11,12]$ to perform both outlier rejection and motion segmentation. This is not strictly motion segmentation as depth discontinuities will also give rise to violation of the affine model.

This paper is arranged as follows. Section 2 contains the notation used. Section 3 details how the drivable region in front of the AGV is recovered. Section 4 shows how independent motions may be segmented if the epipolar constraint is violated. Section 5 shows how progressive dis-occlusion of the road can be used to find independent motions when the epipolar constraint is not violated. Section 6 gives a novel means of collision prediction specific to road going AGVs.

\section{Notation}

Bold face small character $\mathbf{x}$ will refer to homogeneous coordinates of image positions, subscripts $\mathbf{x}_{t}$ will refer to the frame $t$ in which a point is. The homogeneous coordinates are assumed to be in the form $\mathbf{x}=\left(\begin{array}{l}x \\ y \\ f\end{array}\right)$, where $x$ and $y$ are image coordinates and $f$ is in pixels. The projectivity $\mathcal{P}$ describes the motion of the road or ground plane between images. If $\mathbf{x}_{t}$ is a corner on the plane of the road in frame $t$ then it will move to $\mathbf{x}_{t+1}=\mathcal{P} \mathbf{x}_{t}$ in frame $t+1$. The intensity of an image at time $t$ will be given by $I_{t}(\mathbf{x})$.

\section{Dense drivable region detection}

Detection of a drivable region with dense support is divided into two parts. First the planar region segmentation scheme given in [10] is used to segment tracked corners on the road from the background and estimate the projectivity $\mathcal{P}$ that describes the motion of the road between consecutive frames. $\mathcal{P}$ is then used to do a pixel by pixel image difference to give dense support for the drivable region. $\mathcal{P}$ is used to compare corresponding image intensities $I_{t}(\mathbf{x})$ and $I_{t+1}(\mathcal{P} \mathbf{x})$ and if the difference is greater than a value based on an estimate of noise in the image then a given pixel is deemed not to lie in the plane of the road. The sensitivity of this method of dense ground plane detection [10] is such that each pixel is compared with its predicted corresponding pixel and its 6 nearest neighbours.

Figure 1 a) shows the segmentation of tracked corners those in plane of the road are marked with $\oplus$ all other tracked corners are marked with +signs. The drivable region for which dense support exists is shown in figure $1 \mathrm{~b}$ ).

\section{Motion segmentation with violation of the epipolar constraint}

In this section we follow the method of Beardsley [1] for estimating the epipole. This method of motion

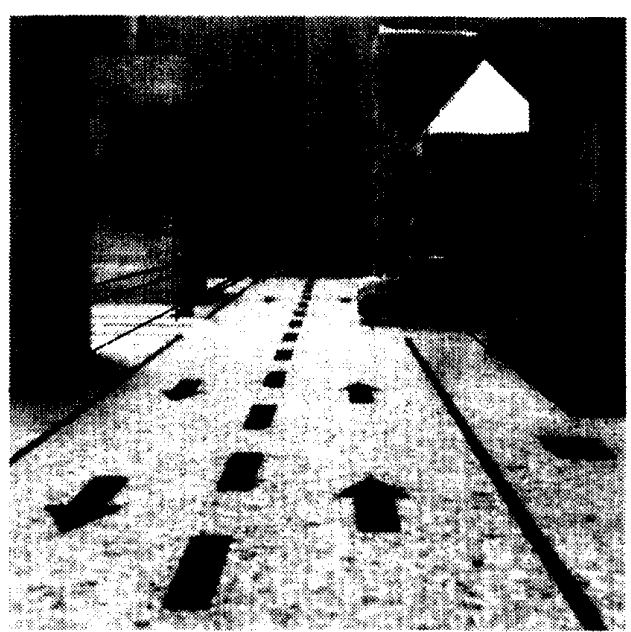

a)

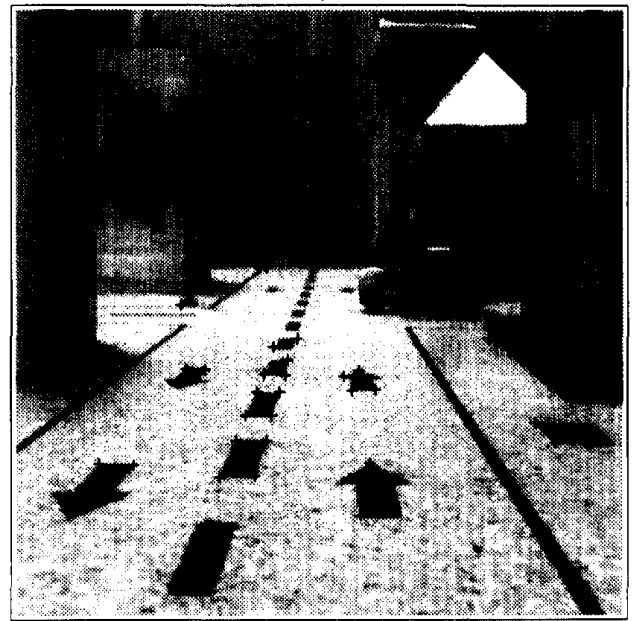

b)

Figure 2: a) an image from a sequence in which independent motion violating the epipolar constraint is present. The vectors superimposed on the image represent the parallax between tracked points and their virtual position on the ground plane. b) the segmentation achieved through grouping points based on their parallax motions. 


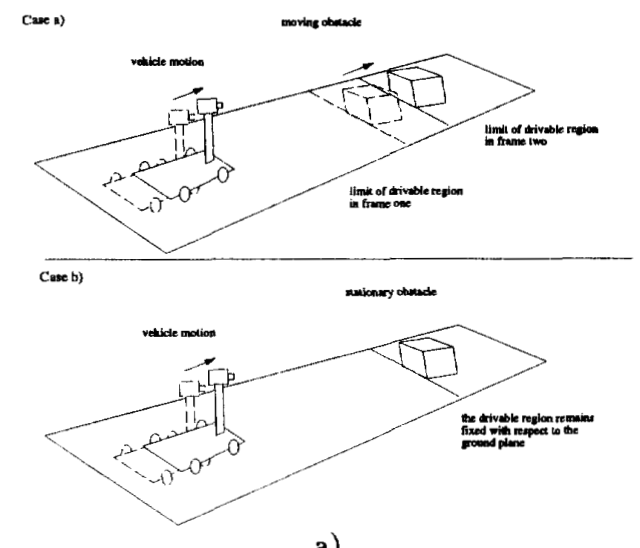

a)

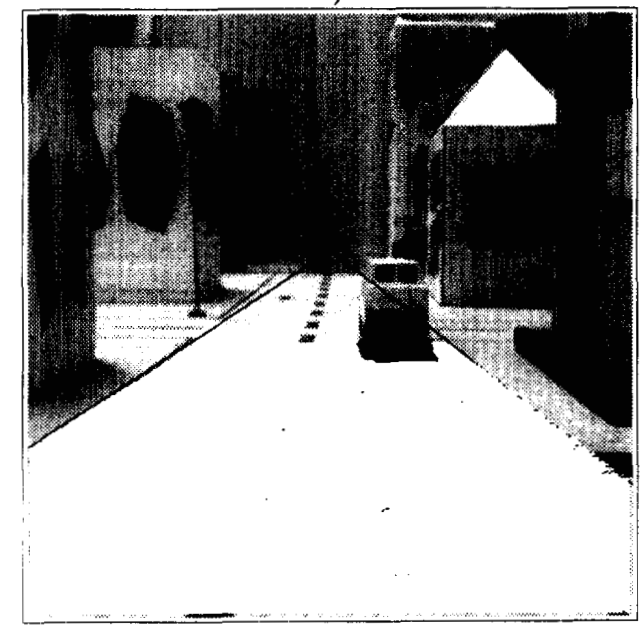

b)

Figure 3: a) the drivable region in front of an $A G V$ will only change if independent motion is occurring. b) white - old drivable region, black - change in drivable region, gray obstacle. The existence of the black region indicates independent motion. estimation depends on the fact that parallax or difference between the motion of a point off the plane and its imagined position on the plane points directly at the epipole, regardless of camera rotation [1]. The advantage of this method is that planes may be reliable tested for and recovered in the presence of noise and independently moving objects [10] and estimation of the epipole then becomes a grouping problem rather than a regression problem. This method also functions without having to solve for scene structure.

Figure 2a) shows an image from a sequence in which independent motion violating the epipolar constraint is present. The vectors superimposed on the image represent the parallax between tracked points and their virtual position on the ground plane. These vectors are grouped according to their points of mutual intersection. The segmentation achieved is displayed in figure 2b) and can be seen to be correct.

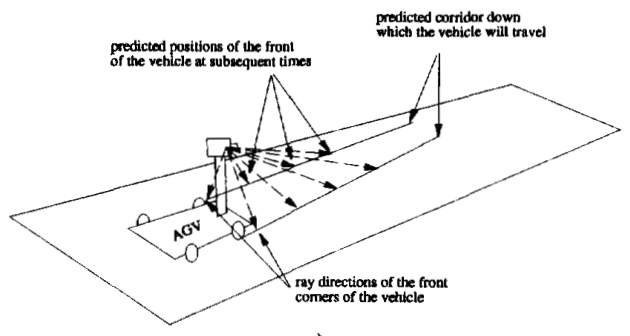

a)

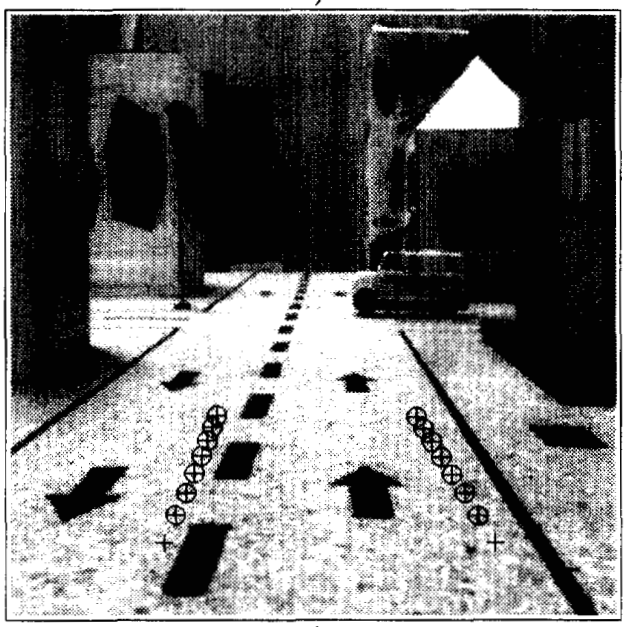

b)

Figure 4: a) the inverse of the projectivity describing the motion of the road is used to 'walk' the front corners of the vehicle forward in time. b) prediction of the positions of the front two corners at future time steps. 


\section{Motion segmentation for parallel motions: anomalous occlusion}

For the case that an AGV is following another vehicle down a straight road the motions of tracked points on the second vehicle do not violate the epipolar constraint. In this case the motion segmentation scheme in section 4 will fail. In order to detect this kind of independent motion it is necessary to look for what might be termed anomalous occlusion, figure 3 . Only if the object is moving will the drivable region in front of the AGV change size. In order to detect this kind of dis-occlusion of the road a minimum of three images are required. The drivable region found between image 2 and 3 must extend further down the plane than the drivable region found between images 1 and 2 . The drivable region found between images 1 and 2 may be mapped into image 3 by applying the projectivity $\mathcal{P}_{2-3}$ to it. Figure 3 illustrates the result of this in practice, the black region on the road is the dis-occluded region found in this way. This kind of progressive dis-occlusion of the road indicates the presence of independent motion.

\section{Collision prediction}

It is well known that flow field divergence may be used to predict time to contact [5] but this suffers the limitation that the object in question must be at the focus of expansion of the moving camera and no account is taken of the rotation of the moving camera. For the case of a camera fixed on an AGV then if camera rotation is present then it is likely that the AGV will be following a curved path, this may well mean that the AGV will miss the obstacle in question, hence rotation is important. Divergence based time to contact collision prediction also fails to embody any information about the size of the AGV.

If the camera mounted on the AGV is fixed with respect to the vehicle then the inverse of the projectivity describing the motion of the road may be used to draw the path in the image that the wheels on the front of the vehicle will take. The ray directions of the front corners of the vehicle must be known in image coordinates (see figure 4). If $\mathbf{l}$ and $\mathbf{r}$ are the ray directions of the left and right front corners of the AGV and the motion of the plane of the road between images $t$ and $t+1$ is described by $\mathcal{P}_{t, t+1}$ then the predicted positions of the front corners of the vehicle will be

$$
\mathbf{r}_{n}=\mathcal{P}_{t, t+1}^{-n} \mathbf{r}
$$

after $n$ time steps and similarly for $\mathbf{l}_{n}$. If the vehicles speed and rotation are constant then the vectors $l_{n}$ and $\mathbf{r}_{n}$ form the sides of the path along which the vehicle will travel projected into image $t+1$. The path may be checked for drivability.

\section{Conclusions}

This paper demonstrates two methods for detecting independent motions for scenarios commonly encountered by AGVs. The first functions when an independently moving object has a velocity which is not parallel to the AGVs. This method has the advantage over existing methods that it is able to distinguish independent motion from occlusion without solving for scene structure. The second technique will detect independently moving objects on the ground plane even if the epipolar constraint is not violated. This technique relies on detecting progressive dis-occlusion of the road. The collision prediction scheme presented is unique in being able to embody an estimate of a vehicles size.

\section{Acknowledgements}

I am grateful to the EC for providing the funding for the HCM program and to Dr A. Blake and Dr R. Mohr for helpful discussions.

\section{References}

[1] P.A. Beardsley, D. Sinclair, and A. Zisserman. Ego-motion from six points. Insight meeting, Catholic University Leuven, 1992.

[2] P.J. Burt and et al. Object tracking with a moving camera. In Proc. 2nd Int. Conf. on Computer Vision, pages $2-12,1989$

[3] B.F. Buxton and D.W. Murray. Optic flow segmentation as an ill-posed and maximum likelihood problem. Image and Vis. Comp., 3,4:163169,1985 .

[4] O.D. Faugeras and S.J. Maybank. Motion from point matches: Multiplicity of solutions. Int. Journal of Computer Vision, 4:225-246, 1988.

[5] J.J. Koenderink and A.J. Van Doorn. Invariant properties of the motion parallax field due to the movement of rigid bodies relative to an observer. Optica Acta, 22(9):773-791, 1975.

[6] H.C Longuet-Higgins. A computer algorithm for reconstructing a scene from two projections. $\mathrm{Na}$ ture, 293(5828):133-135, 1981.

[7] P.F. McLauchlan, I.D. Reid, and D.W. Murray. Coarse motion for saccade control. In Proc. 3rd BMVC, Leeds, 1992.

[8] R.C. Nelson. Qualitative detection of motion by a moving observer. IJCV , 7:1:33-46, 1991.

[9] R.C.Jain. Segmentation of frame sequences obtained by a moving observer. PAMI, 6:624-629 1984

[10] D. Sinclair. Experiments in Motion and Correspondence. PhD thesis, Oxford University, 1993.

[11] S. Smith. Feature based image understanding. D. phil thesis, University of Oxford, 1992.

[12] P. Torr and D. Murray. Outlier detection and motion segmentation. Technical Report OUEL 1987/93, Oxford University, 1993 